\title{
Discordance between oncotype DX recurrence score and RSPC for predicting residual risk of recurrence in ER-positive breast cancer
}

\author{
Andrew Dodson ${ }^{1,2}$ (D) David Okonji ${ }^{3}$ - Laura Assersohn ${ }^{4} \cdot$ Anne Rigg $^{5}$ • \\ Amna Sheri ${ }^{6}$ Nick Turner ${ }^{3} \cdot$ Ian Smith $^{3} \cdot$ Marina Parton $^{3} \cdot$ Mitch Dowsett $^{1,2}$
}

Received: 9 August 2017/ Accepted: 13 September 2017/Published online: 11 November 2017

(c) The Author(s) 2017. This article is an open access publication

\begin{abstract}
Purpose Oncotype DX, a gene expression assay widely employed to aid decision making on adjuvant chemotherapy use in patients with primary oestrogen receptor-positive $(\mathrm{ER}+)$ breast cancer, produces a recurrence score (RS) related to distant disease recurrence (DR) risk (RS\%). In node-negative patients, RS can be integrated with clinicopathological parameters to derive RS-pathology-clinical (RSPC) that improves prognostic accuracy.

Methods Data were collected on patients having clinically indicated tests with an intermediate clinical risk of distant recurrence, and for whom the decision to prescribe chemotherapy remained unclear. Correlation between RS\% and RSPC scores was examined. An agreement table was constructed using risk-categorised data. Association
\end{abstract}

Electronic supplementary material The online version of this article (doi:10.1007/s10549-017-4514-z) contains supplementary material, which is available to authorised users.

Andrew Dodson

andrew.dodson@icr.ac.uk

1 Ralph Lauren Centre for Breast Cancer Research, The Royal Marsden Hospital NHS Foundation Trust, Fulham Road, London SW3 6JJ, UK

2 Institute of Cancer Research, 123 Old Brompton Road, London SW7 3RP, UK

3 Breast Unit, The Royal Marsden Hospital NHS Foundation Trust, Fulham Road, London SW3 6JJ, UK

4 St George's University Hospitals NHS Foundation Trust, London, UK

5 Guy's and St Thomas' Hospitals NHS Foundation Trust, London, UK

6 Royal Free London Hospitals NHS Foundation Trust, London, UK between RS\%-derived categorical risk assignments and treatment recommendation was evaluated.

Results Data on 171 tests (168 patients) were available. Median DR risk by RS\% was $11 \%$ (range 3-34\%), by RSPC it was $15 \%$ (range 4-63\%). Correlation between RS\% and RSPC was $0.702(p<0.001)$. RS\% classified $57.3 \%$ of cases as low-, $32.2 \%$ intermediate- and $10.5 \%$ high-risk for DR; by RSPC proportions were 33.9, 35.7, and $30.4 \%$, respectively. The number of patients receiving chemotherapy recommendations was: $14 / 87(16.1 \%)$ categorised as low-risk by RS\%, $27 / 49(55.1 \%)$ as intermediate-risk and 12/13 (92.3\%) as high-risk. Of 149 patients recommended for endocrine treatment alone, 28 (18.8\%) were categorised by RS\% as low-risk but by RSPC as intermediate- or high-risk.

Conclusions In this group of patients, RSPC assessed fewer patients as low-risk and more as high-risk than did RS\%. The discordances between the scores indicate that RSPC estimates of risk should be considered when selecting patients for endocrine therapy alone.

Keywords Breast cancer - Oncotype DX - Recurrence score $\cdot$ RSPC, prognosis $\cdot$ Recurrence risk

\section{Introduction}

Breast cancer is the most commonly diagnosed invasive cancer in women worldwide. Its incidence rate varies from 19 per 100,000 women in Eastern Africa to 90 per 100,000 women in Western Europe [1]. In the UK, it currently represents $31 \%$ of all new female cancers; there were more than 55,000 newly reported cases in 2015. Incidence is rising and in developed countries is predicted to continue to rise by $2 \%$ per year for at least the next two decades [2]. 
In contrast, breast cancer-specific mortality has fallen dramatically over the past 30-years, with the 10-year survival rate for England and Wales currently standing at around $79 \%$, which greatly improves on the figure of $49 \%$ for the early-1980s [3]; reduced mortality rates are probably due to a combination of earlier detection and improved treatments. In particular, this improvement can be attributed to the introduction of post-surgical (adjuvant) drug treatments, which have made a huge impact in the treatment of oestrogen receptor-positive $(\mathrm{ER}+)$ breast cancer to the extent that more than $85 \%$ of women can expect to remain cancer-free 10-years after their initial diagnosis and treatment. However, it is now clear that, while all such women merit endocrine treatment, not all require additional chemotherapy to prevent recurrence of their disease following surgery. Clinical and pathological parameters carry much information about residual risk of distant disease recurrence (DR), and use of these in systematically produced, evidence-based risk indicators such as the nottingham prognostic index (NPI) has a long history in clinical practice [4].

Molecular tests are increasingly being used to provide information about prognosis to aid decision making about the possibility of omitting chemotherapy when low-risk of distant DR is indicated [5]. Oncotype DX Breast Cancer Assay is such a molecular test, commercially provided from genomic health, incorporated (GHI). It produces a recurrence score (RS) for the purpose of prognostication. In the England and Wales, in 2013, it was recommended by the National Institute for Health and Care Excellence (NICE) for use in patients with ER+, human epidermal receptor-2-negative (HER2-) early advanced breast cancer, assessed on the basis of clinical algorithms to be at intermediate-risk of DR and who would benefit from additional prognostic information to help guide chemotherapy prescribing [6]. It has been funded by NHS England for patients that have node-negative disease since 2015, and are judged by standard clinical and pathological criteria to be at intermediate-risk of distant disease recurrence, and where the decision to prescribe chemotherapy remains unclear.

Tang and colleagues have examined the information provided by RS combined with that derived from traditional clinicopathological features (tumour grade and size and patient age) standardised against endocrine treatment type (tamoxifen or aromatase inhibitor) [7]. Using data derived from node-negative patients entered into the tamoxifen-treatment arm of the National Surgical Adjuvant Breast and Bowel (NSABP) B-14 study [8], and the monotherapy-treatment arms of the translational research cohort in the Arimidex, Tamoxifen Alone or in Combination (TransATAC) study [9], found that the information contained in the two measures were complementary, and that combining them produced an indicator having substantially more prognostic power than either set used singly: they termed this integrated risk estimate RSpathology-clinical (RSPC). RSPC risk estimates may be derived by means of a web-based tool provided for educational purposes by GHI, which is freely accessible to clinicians [10]. However, few oncologists use RSPC in patient management.

Use of the Oncotype DX RS test is increasing in many countries, and is presently, standard practice in appropriate cases within most NHS clinical breast oncology units in England and Wales. We combined the data from four NHS Breast Units within the London region to compare risk estimates provided by RS and RSPC and to assess the relationship with RS-based clinical recommendations for chemotherapy use.

\section{Materials and methods}

Anonymised data (RS, patient age at surgery, tumour maximum diameter measured at resection, tumour grade, menopausal status, planned endocrine-based treatment type, and final clinical recommendation on addition of chemotherapy) were collected from four London NHS Foundation Trusts (Guy's and St Thomas', Royal Free London, The Royal Marsden and St George's). Data were collected on patients having Oncotype DX tests ordered between January 2015 and September 2016 as part of routine clinical care in-line with NHS England agreed use in patients at intermediate-risk of distant recurrence as assessed using NPI (score $>3.40$ and $\leq 5.40$ ) or PREDICT (score $\geq 3 \%$ benefit). In the case of The Royal Marsden, this was a consecutive series of all patients tested; for the other three centres, the patient series included only those where there was documented evidence of a clinical recommendation following receipt of the RS result.

The Oncotype DX breast cancer assay reports two measures related to recurrence risk: the RS itself, and an RS-derived percentage estimate of residual 10-year risk of distant DR assuming 5-years' adjuvant endocrine treatment with tamoxifen, termed RS\%. Both RS\% and RSPC produce results on a $0-100 \%$ scale, and both use the same cutpoints to define risk boundaries (vide infra), therefore to maximise comparability, we report here on a comparison between RS\% and RSPC.

The published risk categories for RS were originally chosen based on results from the tamoxifen-treatment arm of the NSAPB B-20 trial [8], with scores less than 18 designating low-risk, those between 18 and 30 intermediate-risk, and those above 30 high-risk. On the basis of results from the NSAPB B-14 study, which was used to validate the assay, an RS of 18 equates to a 10-year 
residual risk of approximately $12 \%$ in a tamoxifen-treated population, while an $\mathrm{RS}$ of 31 is equivalent to a $21 \%$ risk. The same risk cut-points were designated for RSPC by Tang et al. [7] in their paper, and they have been retained here.

RS\% was calculated using tamoxifen as the intended endocrine treatment, which is in-line with the default treatment for GHI-reported clinical cases. Tumour size and grade, patient age at surgery and intended endocrine treatment (tamoxifen or aromatase inhibitor) were combined with the RS to produce an RSPC. Web-based tools provided by GHI were used to calculate the $\mathrm{RS} \%$ and RSPC scores for each study case [10].

The NPI was calculated for each case using the published formula:

$\mathrm{NPI}=[0.2 \times S]+N+G$

[where $S$ is the size of the index lesion in centimetres, $N$ is the node status $(0$ nodes $=1,1-4$ nodes $=2,>4$ nodes $=3$ ), $\mathrm{G}$ is the grade of tumour (Grade $\mathrm{I}=1$, Grade $\mathrm{II}=2$, Grade III = 3)] [11].

Correlation between RS\% and RSPC scores was assessed using Spearman's rho statistic. RS\% and RSPC data were log-transformed prior to analysis. Agreement tables were constructed to compare the agreement of categorical assignments made by RS\% and RSPC. RS\% and RSPC derived categorical scores were correlated with chemotherapy recommendation.

\section{Results}

\section{Study cases}

A total of 177 cases from 174 patients having tumours tested by the Oncotype DX assay were submitted by the four hospitals' breast units during the study period (3 patients each had 2 concurrent assays performed on discrete tumours or tumour foci); 6 cases (6 patients) were excluded from analysis, 1 due to node-positive status and 5 due to missing RS result. Centre 3 contributed just under $55 \%$ of the cases, Centre 1 approximately $20 \%$ and Centres 2 and 4 approximately $12 \%$ each. $171 / 177$ cases $(96.6 \%)$ from 168 patients $(96.2 \%)$ were analysed for concordance.

An additional of 19 cases (19 patients) were excluded from analysis of treatment recommendation due to unavailable recommendation data; therefore 152 cases (149 patients) were available. Supplementary Table S1 tabulates study and centre-specific case and patient numbers.

Median patient age at surgery was 53 years (range 24-78 years), with pre- and post-menopausal women being almost equally represented (46.6 and $49.4 \%$ respectively). Patient demographics showed some centre-to-centre variation; the median age for women in Centre 1's case-set was 49 years (range 33-74 years), noticeably lower than that for the study overall; at this centre, the proportion of pre-menopausal women was also higher (64.9\%). These contrasts with Centre 3, where the median age of its patients was 56 years (range $24-78$ years) and $40.4 \%$ were pre-menopausal.

Almost all patients were node-negative (94.8\%), in-line with NHS England referring guidance for the test. Seven patients $(4.0 \%)$ presented with one or more micro-metastasis and have been classified as node-negative. The single node-positive patient has been excluded.

Median tumour diameter was $23 \mathrm{~mm}$ (range 6-120 mm), with the median diameter for cases from Centres 2, 3 and 4 all being closely similar to this; in contrast, tumours referred for testing at Centre 1 tended to be smaller, with a median tumour diameter of $18 \mathrm{~mm}$ (range 6-70 $\mathrm{mm}$ ).

Tumour grade distribution was closely similar for all centres, with the majority being Grade 2 (58.2\%) or Grade $3(40.1 \%)$, only Centre 3 tested any tumours that were Grade $1(1.1 \%)$.

Median and range of NPI was closely similar for all centres. For the whole study, the median was 4.0 (range 2.4-5.4). Similarity in results for this index between centres indicates that although the centre-specific differences existed in the distributions of tumour size and grade, these characteristics were balanced for individual patients.

Patient demographics and tumour characteristics together with information on NPI and treatment recommendations are shown in Table 1.

\section{RS\% and RSPC scores}

Median 10-year DR risk estimate by RSPC was $15 \%$ (range 4-63\%); it was appreciably lower by $\mathrm{RS} \%$ (11\%, range 3-34\%) (Table 1). Correlation between RS\% and RSPC was statistically significant $(r h o=0.702, p<0.001$, twotailed), with the rho value indicating that just under half of the variation seen in one measure could be explained by variation in the other.

\section{Agreement analysis for categorical assignment}

Cross-tabulation comparison demonstrated evidence for a considerable non-concordance between RS\%- and RSPCbased risk categorisations. The proportions of cases assigned respectively to each of the three risk categories by $\mathrm{RS} \%$ and RSPC were markedly disparate. When RS\% was used to classify cases $57.3 \%$ were designated as low-, $32.2 \%$ as intermediate-, and $10.5 \%$ as high-risk. When RSPC was used, the figures were, 33.9, 35.7 and 30.4\%, respectively (see Chart 1 in Fig. 1). 
Table 1 Patient demographics and tumour characteristics

\begin{tabular}{|c|c|c|c|c|c|}
\hline & All centres & Centre 1 & Centre 2 & Centre 3 & Centre 4 \\
\hline Gender & $N$ patients $(\%)$ & & & & \\
\hline Female & $171(98.3 \%)$ & $37(100.0 \%)$ & $19(95.0 \%)$ & $92(97.9 \%)$ & $23(100.0 \%)$ \\
\hline Male & $2(1.1 \%)$ & $0(0 \%)$ & $1(5.0 \%)$ & $1(1.1 \%)$ & $0(0 \%)$ \\
\hline Unknown & $1(0.6 \%)$ & $0(0 \%)$ & $0(0 \%)$ & $1(1.1 \%)$ & $0(0 \%)$ \\
\hline Age at surgery (years) & Median (range) & & & & \\
\hline Age at surgery (years) & $53(24-78)$ & $49(33-74)$ & $51(37-72)$ & $56(24-78)$ & $54(38-74)$ \\
\hline Unknown ( $N$ patients $(\%))$ & $1(0.6 \%)$ & $0(0 \%)$ & $0(0 \%)$ & $1(1.1 \%)$ & $0(0 \%)$ \\
\hline Menopausal status & $N$ patients $(\%)$ & & & & \\
\hline Pre-menopausal & $81(46.6 \%)$ & $24(64.9 \%)$ & $8(40.0 \%)$ & $38(40.4 \%)$ & $11(47.8 \%)$ \\
\hline Post-menopausal & $86(49.4 \%)$ & $13(35.1 \%)$ & $10(50.0 \%)$ & $51(54.3 \%)$ & $12(52.2 \%)$ \\
\hline Peri-menopausal & $2(1.1 \%)$ & $0(0 \%)$ & $1(5.0 \%)$ & $1(1.1 \%)$ & $0(0 \%)$ \\
\hline Not applicable (male) & $2(1.1 \%)$ & $0(0 \%)$ & $1(5.0 \%)$ & $1(1.1 \%)$ & $0(0 \%)$ \\
\hline Unknown & $3(1.7 \%)$ & $0(0 \%)$ & $0(0 \%)$ & $3(3.2 \%)$ & $0(0 \%)$ \\
\hline Number of involved nodes & $N$ patients $(\%)$ & & & & \\
\hline$N=0$ & $165(94.8 \%)$ & $35(94.6 \%)$ & $20(100.0 \%)$ & $88(93.6 \%)$ & $22(95.6 \%)$ \\
\hline$N \geq 1$ & $1(0.6 \%)$ & $0(0 \%)$ & $0(0 \%)$ & $0(0 \%)$ & $1(4.3 \%)$ \\
\hline Micro-metastasis & $7(4.0 \%)$ & $2(5.4 \%)$ & $0(0 \%)$ & $5(5.3 \%)$ & $0(0 \%)$ \\
\hline Unknown & $1(0.6 \%)$ & $0(0 \%)$ & $0(0 \%)$ & $1(1.1 \%)$ & $0(0 \%)$ \\
\hline Tumour diameter (mm) & Median (range) & & & & \\
\hline Tumour diameter & $23(6-120)$ & $18(6-70)$ & $25.5(9-42)$ & $25(10-120)$ & $20(6-56)$ \\
\hline \multicolumn{6}{|l|}{$N$ cases $(\%)$} \\
\hline$<10 \mathrm{~mm}$ & $6(3.4 \%)$ & $3(8.1 \%)$ & $1(5.0 \%)$ & $0(0 \%)$ & $2(8.7 \%)$ \\
\hline $10-19$ & $46(26.0 \%)$ & $20(54.1 \%)$ & $4(20.0 \%)$ & $16(17.0 \%)$ & $6(26.1 \%)$ \\
\hline $20-29$ & $62(35.0 \%)$ & $9(24.3 \%)$ & $8(40.0 \%)$ & $34(36.2 \%)$ & $11(47.8 \%)$ \\
\hline $30-39$ & $27(15.3 \%)$ & $2(5.4 \%)$ & $6(30.0 \%)$ & $16(17.0 \%)$ & $3(13.0 \%)$ \\
\hline $40-49$ & $17(9.6 \%)$ & $3(8.1 \%)$ & $1(5.0 \%)$ & $12(12.8 \%)$ & $1(4.3 \%)$ \\
\hline$\geq 50$ & $18(10.2 \%)$ & $2(5.4 \%)$ & $0(0 \%)$ & $15(16.0 \%)$ & $1(4.3 \%)$ \\
\hline Unknown & $1(0.6 \%)$ & $0(0 \%)$ & $0(0 \%)$ & $1(1.1 \%)$ & $0(0 \%)$ \\
\hline Tumour grade & Median (range) & & & & \\
\hline Tumour grade & $2(1-3)$ & $2(2-3)$ & $2(2-3)$ & $2(1-3)$ & $2(2-3)$ \\
\hline \multicolumn{6}{|l|}{$\mathrm{N}$ cases $(\%)$} \\
\hline Grade 1 & $2(1.1 \%)$ & $0(0 \%)$ & $0(0 \%)$ & $2(2.1 \%)$ & $0(0 \%)$ \\
\hline Grade 2 & $103(58.2 \%)$ & $23(59.0 \%)$ & $11(55.0 \%)$ & $53(56.4 \%)$ & $15(65.2 \%)$ \\
\hline Grade 3 & $71(40.1 \%)$ & $16(41.0 \%)$ & $9(45.0 \%)$ & $38(40.4 \%)$ & $8(22.7 \%)$ \\
\hline Unknown & $1(0.6 \%)$ & $0(0 \%)$ & $0(0 \%)$ & $1(1.1 \%)$ & $0(0 \%)$ \\
\hline Prognostic indices & Median (range) & & & & \\
\hline NPI & $4.0(2.4-5.4)$ & $3.8(3.1-4.9)$ & $3.7(3.4-4.8)$ & $4.1(2.4-5.4)$ & $3.7(3.1-4.7)$ \\
\hline $\mathrm{RS} \%$ & $11 \%(3-34 \%)$ & $11 \%(4-34 \%)$ & $10 \%(3-30 \%)$ & $11 \%(3-34 \%)$ & $9 \%(5-23 \%)$ \\
\hline RSPC & $15 \%(4-63 \%)$ & $14 \%(4-63 \%)$ & $14 \%(5-33 \%)$ & $18 \%(5-51 \%)$ & $13(6-31 \%)$ \\
\hline
\end{tabular}

Percentages indicate the proportions of patients for age, menopausal status and number of involved nodes; for tumour size and grade percentages they are the proportions of cases. NPI, RS\% and RSPC distributions for the whole study and for each centre individually are also given

Agreement was seen in a total of $99 / 171$ cases $(57.9 \%)$; $5(2.9 \%)$ had a lower risk category classification and 67 (39.2\%) a higher classification when RSPC rather than RS\% was used. Comparing the categorical assignment of cases made by RS\% and RSPC in more detail; 32 (18.7\%) classified as low-risk by RS\% were increased by RSPC to intermediate-risk, and 12 (7.0\%) to high-risk; 23 (13.5\%) classified as intermediate-risk by RS\% were increased to high-risk by RSPC. In contrast, decreased risk-classification of cases by RSPC compared to RS\% was seen in only 
Fig. 1 (Combined figure) Chart: The histogram shows cross-classification of cases by RS\% and RSPC to each risk category. Case numbers were similar for RS\% and RSPC with respect to intermediate-risk; however, the number of cases in the low-risk category was very noticeably higher when RS\% was used to assign the risk compared to RSPC, and concomitantly, the number designated as high-risk very noticeably lower. Table: Contingency table for risk category assignment. Agreement data are shown for all cases in the study and individually for centre-specific cases

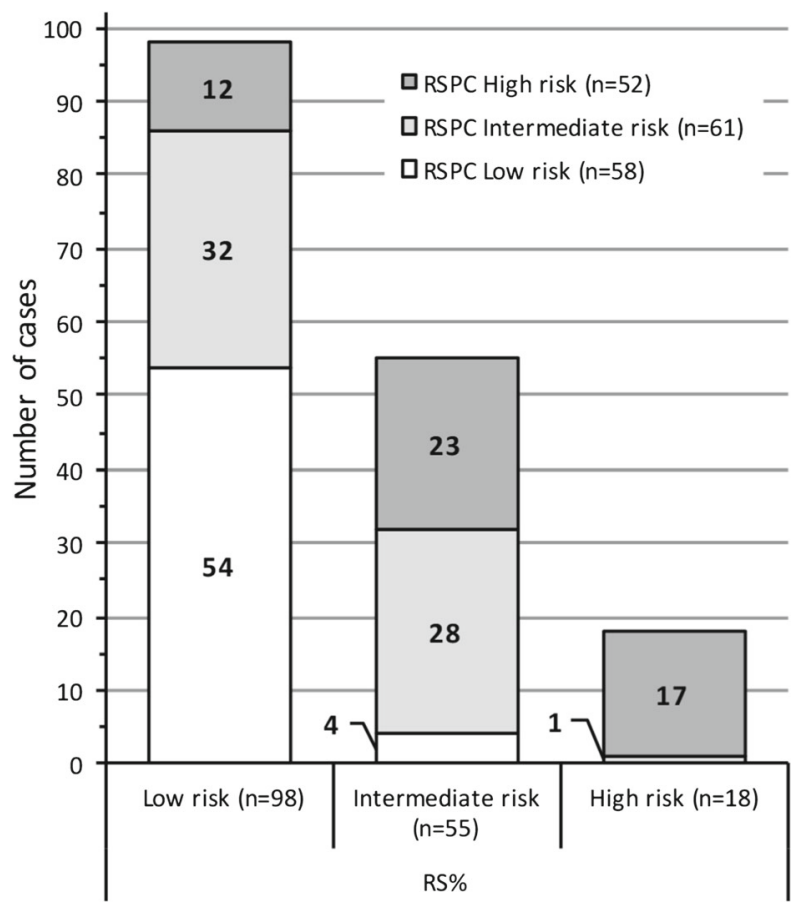

\begin{tabular}{|c|c|c|c|c|c|}
\hline & \multicolumn{3}{|c|}{ Risk assigned by RS\% } & \multirow{2}{*}{ Totals } \\
\hline & & Low & Intermediate & High & \\
\hline \multicolumn{6}{|c|}{ All cases } \\
\hline \multirow{24}{*}{$\begin{array}{c}\text { Risk } \\
\text { assigned } \\
\text { by RSPC }\end{array}$} & Low & $54(31.6 \%)$ & $4(2.3 \%)$ & $0(0 \%)$ & $58(33.9 \%)$ \\
\hline & Intermediate & $32(18.7 \%)$ & $28(16.4 \%)$ & $1(0.6 \%)$ & $61(35.7 \%)$ \\
\hline & High & $12(7.0 \%)$ & $23(13.5 \%)$ & 17 (9.9\%) & $52(30.4 \%)$ \\
\hline & Totals & $98(57.3 \%)$ & $55(32.2 \%)$ & $18(10.5 \%)$ & 171 (100\%) \\
\hline & \multicolumn{5}{|c|}{ Centre 1} \\
\hline & Low & 15 (40.5\%) & $2(5.4 \%)$ & $0(0 \%)$ & $17(45.9 \%)$ \\
\hline & Intermediate & $5(13.5 \%)$ & $6(16.2 \%)$ & $0(0 \%)$ & $11(29.7 \%)$ \\
\hline & High & $2(5.4 \%)$ & $3(8.1 \%)$ & $4(10.8 \%)$ & $9(24.3 \%)$ \\
\hline & Totals & $22(59.5 \%)$ & $11(29.7 \%)$ & $4(10.8 \%)$ & 37 (100\%) \\
\hline & \multicolumn{5}{|c|}{ Centre 2} \\
\hline & Low & $8(40.0 \%)$ & $0(0 \%)$ & $0(0 \%)$ & $8(40.0 \%)$ \\
\hline & Intermediate & $6(30.0 \%)$ & $3(15.0 \%)$ & $0(0 \%)$ & $9(45.0 \%)$ \\
\hline & High & $0(0 \%)$ & $0(0 \%)$ & $3(15 \%)$ & $3(15.0 \%)$ \\
\hline & Totals & $14(70.0 \%)$ & $3(15.0 \%)$ & $3(15.0 \%)$ & $20(100 \%)$ \\
\hline & \multicolumn{5}{|c|}{ Centre 3} \\
\hline & Low & $23(25.3 \%)$ & $2(2.2 \%)$ & $0(0 \%)$ & $25(27.5 \%)$ \\
\hline & Intermediate & $16(17.6 \%)$ & $13(14.3 \%)$ & $1(1.1 \%)$ & $30(33.0 \%)$ \\
\hline & High & $9(9.9 \%)$ & $18(19.8 \%)$ & 9 (9.9\%) & 36 (39.6\%) \\
\hline & Totals & $48(52.7 \%)$ & $33(36.3 \%)$ & $10(11 \%)$ & $91(100 \%)$ \\
\hline & \multicolumn{5}{|c|}{ Centre 4} \\
\hline & Low & 8 (34.8\%) & $0(0 \%)$ & $0(0 \%)$ & $8(34.8 \%)$ \\
\hline & Intermediate & 5 (21.7\%) & 6 (26.1\%) & $0(0 \%)$ & 11 (47.8\%) \\
\hline & High & $1(4.3 \%)$ & $2(8.7 \%)$ & $1(4.3 \%)$ & 4 (17.4\%) \\
\hline & Totals & 14 (60.9\%) & $8(34.8 \%)$ & 1 (4.3\%) & 23 (100\%) \\
\hline
\end{tabular}


5 cases, 4 (2.3\%) decreased from intermediate-risk to lowrisk, and $1(0.6 \%)$ changed from high-risk to intermediaterisk (see Table in Fig. 1).

\section{Treatment recommendation}

Data on treatment recommendations were available on $149 / 174$ patients $(85.6 \%)$ (Table 2). Overall, within the study, 96 patients $(64.4 \%)$ were recommended to receive endocrine treatment alone, while there was a recommendation made for adding chemotherapy to endocrine-based treatment for 53 patients $(35.6 \%)$. There were centrespecific differences seen in the proportions of patients receiving recommendations for the addition of chemotherapy; at Centre 1, the proportion of patients recommended to receive chemotherapy closely matched that of the whole study, while Centres 2 and 4 had similar proportion to each other, both of which were substantially lower than that in the whole study. In contrast, chemotherapy was recommended to a higher proportion of patients whose tests were conducted as part of their care pathway at Centre 3 (for details see Table 2).

Stratified by RS\%-designated risk category, proportions of patients having a recommendation for the addition of chemotherapy were, $14 / 87(16.1 \%)$ in the low-risk category, $27 / 49(55.1 \%)$ in the intermediate-risk category, and $12 / 13(92.3 \%)$ in the high-risk category. These results clearly indicate an association between RS\% result and treatment recommendation, and this association was recapitulated in the individual data from all centres (Table 2).

A substantial number of patients with low-risk indications by RS\% but RSPC risk scores indicative of intermediate- or high-risk received recommendations for adjuvant endocrine-based therapy alone. There was a total of 28 such patients $(18.8 \%), 22(14.8 \%)$ with an intermediate-risk and $6(4.0 \%)$ with a high-risk indication by RSPC. Such patients were present in the cohorts submitted by each centre (Table 3 ).

\section{Discussion}

We report on a comparison between RS\%, which is an RSderived percentage estimate and RSPC, which incorporates clinicopathological parameters. Both produce results on a $0-100 \%$ scale, indicative of residual 10-year risk of distant DR assuming 5-years' adjuvant endocrine treatment with tamoxifen using the same cut-points to define risk boundaries.

In the study by Tang et al., a significant improvement in prognostic performance was seen for RSPC over RS [7]. Similarly, RSPC had substantially improved prognostic performance when compared to clinicopathologic features

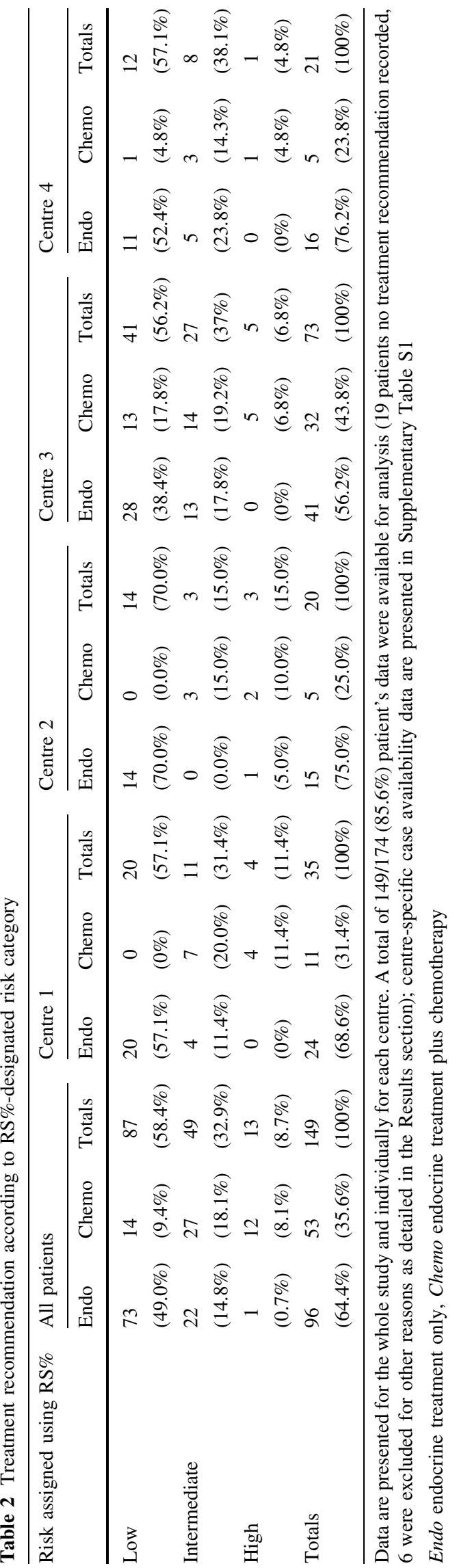


Table 3 Cross-tabulation table for treatment recommendations according to RS\% and RSPC designated risk category

\begin{tabular}{|c|c|c|c|c|c|c|c|c|}
\hline & \multicolumn{6}{|c|}{ Risk assigned by RS\% } & \multirow{2}{*}{\multicolumn{2}{|c|}{ Totals }} \\
\hline & \multicolumn{2}{|l|}{ Low } & \multicolumn{2}{|l|}{ Intermediate } & \multicolumn{2}{|l|}{ High } & & \\
\hline & Endo & Chemo & Endo & Chemo & Endo & Chemo & Endo & Chemo \\
\hline \multicolumn{9}{|c|}{ Risk assigned by RSPC } \\
\hline \multicolumn{9}{|l|}{ All patients } \\
\hline Low & $45(30.2 \%)$ & $5(3.4 \%)$ & $2(1.3 \%)$ & $1(0.7 \%)$ & $0(0 \%)$ & $0(0 \%)$ & $47(31.5 \%)$ & $6(4.0 \%)$ \\
\hline Intermediate & $22(14.8 \%)$ & $6(4.0 \%)$ & $14(9.4 \%)$ & $11(7.4 \%)$ & $0(0 \%)$ & $1(0.7 \%)$ & $36(24.2 \%)$ & $18(12.1 \%)$ \\
\hline High & $6(4.0 \%)$ & $3(2.0 \%)$ & $6(4.0 \%)$ & $15(10.1 \%)$ & $1(0.7 \%)$ & $11(7.4 \%)$ & $13(8.7 \%)$ & $29(19.5 \%)$ \\
\hline Totals & $73(49.0 \%)$ & $14(9.4 \%)$ & $22(14.8 \%)$ & $27(18.1 \%)$ & $1(0.7 \%)$ & $12(8.1 \%)$ & $96(64.4 \%)$ & $53(35.6 \%)$ \\
\hline \multicolumn{9}{|l|}{ Centre 1} \\
\hline Low & $13(37.1 \%)$ & $0(0 \%)$ & $2(5.7 \%)$ & $0(0 \%)$ & $0(0 \%)$ & $0(0 \%)$ & $15(42.9 \%)$ & $0(0 \%)$ \\
\hline Intermediate & $5(14.3 \%)$ & $0(0 \%)$ & $2(5.7 \%)$ & $4(11.4 \%)$ & $0(0 \%)$ & $0(0 \%)$ & $7(20 \%)$ & $4(11.4 \%)$ \\
\hline High & $2(5.7 \%)$ & $0(0 \%)$ & $0(0 \%)$ & $3(8.6 \%)$ & $0(0 \%)$ & $4(11.4 \%)$ & $2(5.7 \%)$ & $7(20 \%)$ \\
\hline Totals & $20(57.1 \%)$ & $0(0 \%)$ & $4(11.4 \%)$ & $7(20 \%)$ & $0(0 \%)$ & $4(11.4 \%)$ & $24(68.6 \%)$ & $11(31.4 \%)$ \\
\hline \multicolumn{9}{|l|}{ Centre 2} \\
\hline Low & $8(40 \%)$ & $0(0 \%)$ & $0(0 \%)$ & $0(0 \%)$ & $0(0 \%)$ & $0(0 \%)$ & $8(40 \%)$ & $0(0 \%)$ \\
\hline Intermediate & $6(30 \%)$ & $0(0 \%)$ & $0(0 \%)$ & $3(15 \%)$ & $0(0 \%)$ & $0(0 \%)$ & $6(30 \%)$ & $3(15 \%)$ \\
\hline High & $0(0 \%)$ & $0(0 \%)$ & $0(0 \%)$ & $0(0 \%)$ & $1(5 \%)$ & $2(10 \%)$ & $1(5 \%)$ & $2(10 \%)$ \\
\hline Totals & $14(70 \%)$ & $0(0 \%)$ & $0(0 \%)$ & $3(15 \%)$ & $1(5 \%)$ & $2(10 \%)$ & $15(75 \%)$ & $5(25 \%)$ \\
\hline \multicolumn{9}{|l|}{ Centre 3} \\
\hline Low & $16(21.9 \%)$ & $5(6.8 \%)$ & $0(0 \%)$ & $1(1.4 \%)$ & $0(0 \%)$ & $0(0 \%)$ & $16(21.9 \%)$ & $6(8.2 \%)$ \\
\hline Intermediate & $9(12.3 \%)$ & $5(6.8 \%)$ & $7(9.6 \%)$ & $3(4.1 \%)$ & $0(0 \%)$ & $1(1.4 \%)$ & $16(21.9 \%)$ & $9(12.3 \%)$ \\
\hline High & $3(4.1 \%)$ & $3(4.1 \%)$ & $6(8.2 \%)$ & $10(13.7 \%)$ & $0(0 \%)$ & $4(5.5 \%)$ & $9(12.3 \%)$ & $17(23.3 \%)$ \\
\hline Totals & $28(38.4 \%)$ & $13(17.8 \%)$ & $13(17.8 \%)$ & $14(19.2 \%)$ & $0(0 \%)$ & $5(6.8 \%)$ & $41(56.2 \%)$ & $32(43.8 \%)$ \\
\hline \multicolumn{9}{|l|}{ Centre 4} \\
\hline Low & $8(38.1 \%)$ & $0(0 \%)$ & $0(0 \%)$ & $0(0 \%)$ & $0(0 \%)$ & $0(0 \%)$ & $8(38.1 \%)$ & $0(0 \%)$ \\
\hline Intermediate & $2(9.5 \%)$ & $1(4.8 \%)$ & $5(23.8 \%)$ & $1(4.8 \%)$ & $0(0 \%)$ & $0(0 \%)$ & $7(33.3 \%)$ & $2(9.5 \%)$ \\
\hline High & $1(4.8 \%)$ & $0(0 \%)$ & $0(0 \%)$ & $2(9.5 \%)$ & $0(0 \%)$ & $1(4.8 \%)$ & $1(4.8 \%)$ & $3(14.3 \%)$ \\
\hline Totals & $11(52.4 \%)$ & $1(4.8 \%)$ & $5(23.8 \%)$ & $3(14.3 \%)$ & $0(0 \%)$ & $1(4.8 \%)$ & $16(76.2 \%)$ & $5(23.8 \%)$ \\
\hline
\end{tabular}

A total of 149/174 (85.6\%) patient's data were available for analysis (19 patients no treatment recommendation recorded, 6 were excluded for other reasons as detailed in the Results section); centre-specific case availability data are presented in Supplementary Table S1. Figures highlighted in bold indicate cases that might be considered to represent the risk category discrepant cases that have the most potential clinical impact

alone. Here, we show that there is a substantial upward shift in risk categorisation when RSPC rather than RS\% score is applied to a contemporary cohort of patients. Considering the effect on treatment recommendations, the most clinically relevant changes are those that cause 37 patients $(24.8 \%)$ to cross from low- to intermediate-risk, or, low- to high-risk. In this group, 28 (75.7\%) received an endocrine only recommendation, which they may not have if the RSPC result had been used in place of RS\%. Figure 2(c) shows a scatterplot relating all RS\% and RSPC scores. In it, most points clearly lie above the line of equivalence, indicating that even when they do not cross risk boundaries, the indicated risk of DR by is higher by RSPC compared to RS\%.
In patients where RSPC increased risk category assignment, median tumour size was greater than for the whole study population ( 30 vs. $23 \mathrm{~mm}$ ) and seems to have been the main driver of the increase in risk estimation by RSPC since other demographic and clinicopathological parameters did not differ. Consequently, median NPI was also higher, at 4.3 versus 4.0 .

The trend for RSPC results within our series to indicate an increased risk of distant DR as in direct contrast with that seen by Tang et al. in the NSABP B-14 and TransATAC cohorts, where the tendency was RSPC results to classify fewer patients than RS as intermediate-risk (17.8 vs. $26.7 \%)$ and more patients as lower risk (63.8 vs. $54.2 \%)$. This difference can be explained by the variances in the distributions of patient age, tumour size and most 

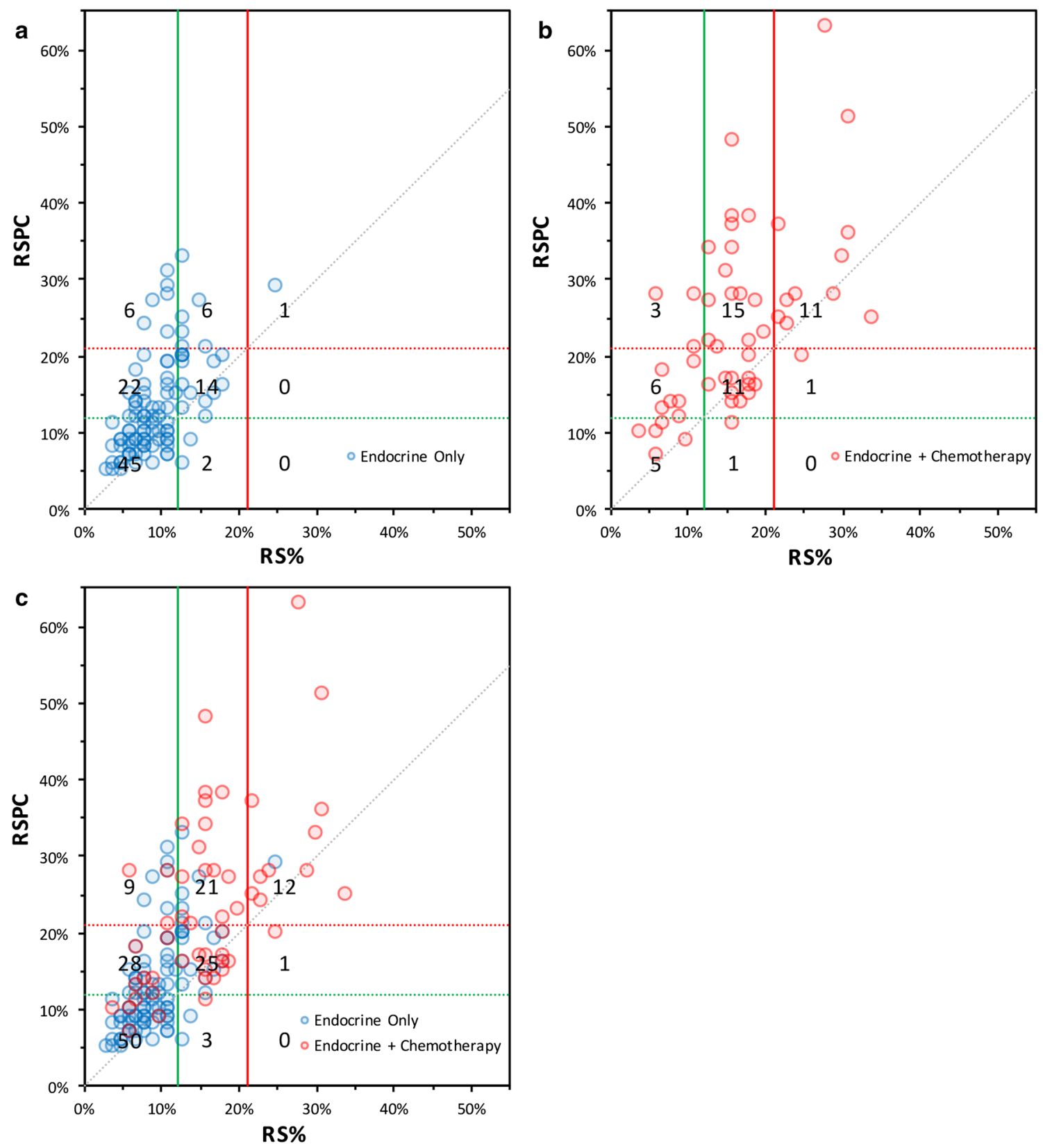

Fig. 2 Composite figure comprising three scatterplots showing the position of each patient with regard to their RS\% and RSPC scores. a shows patients for whom the recommendation was for endocrine treatment alone, indicated by blue circles at the intersection of the RS\% and RSPC scores for that patient's tumour. b shows patients in whom the recommendation was for the addition of chemotherapy to endocrine treatment, indicated by red circles. c this scatterplot overlays both sets of cases as shown (a) and (b). In all plots, the green

particularly tumour grade that exist in the populations examined by Tang et al. and in our study. The median ages in the B-14, TransATAC and our study were 58, 63 and 53 years, respectively; median tumour sizes were 20,18 and $23 \mathrm{~mm}$; the proportions of Grade 1 tumours were 35 , 22 and $1 \%$, while those for Grade 3 tumours were 20, 18

lines indicate boundaries between low-risk and intermediate-risk categories, while the red lines indicate those between intermediaterisk and high-risk; solid lines are applicable to RS\% scores, dottedlines to RSPC scores. The grey dotted line indicates the line of equality. The numbers in each sector are number of patients (note that due to overlying data points, the number of points in the sector may not appear to agree with the figure shown)

and $40 \%$. All these measures indicate that the patients within our study represented a population at higher risk, this being an inevitable consequence of eligibility requirements set for the test by NHS England.

Limited evidence has been published indicating that patients with low Oncotype DX scores may gain little from 
chemotherapy even though their clinicopathological features may indicate they are at high-risk of recurrence $[12,13]$. However, central meta-analyses of chemotherapy (anthracycline- or taxane-based) trials in both ER+ and ER- breast cancer patients have produced little or no evidence for stratification of benefit according to clinicopathological features. Rather, they have shown that the benefit from chemotherapy is proportional to overall risk [14]. With the current discordant data, it is unknown if the superior prognostic performance of RSPC compared to RS, it would necessarily translate into improved prediction of benefit from chemotherapy. The data in this current study cannot address the relative predictive value of either score. In particular, it is cannot be known if patients who are converted from low-risk RS to intermediate-risk by RSPC, derive benefit from chemotherapy.

The TAILORx prospective trial examining the use of Oncotype DX scores to stratify treatment in ER+ (and/or progesterone receptor-positive), HER2-, node-negative early advanced breast cancer has reported 5-year results on its low-risk patient cohort assigned to receive endocrine treatment alone [15]. In this group of 1626 patients, the rate of freedom from DR was 99.3\% (95\% CI 98.7-99.6). Potentially, this is very strong evidence for the effectiveness of the RS alone in assigning risk. However, the eligibility criteria for patients in TAILORx differ very substantially from those in our study; the RS cut-off defining low-risk was $\leq 10$ and not $\leq 17$, tumours could be up to $50 \mathrm{~mm}$ in diameter only if Grade 1 , and $\leq 10 \mathrm{~mm}$ if Grade 2 or 3. Examination of the data of size distribution for the study shows that the vast majority (92\%) were less than $30 \mathrm{~mm}$. In summary, this population's clinicopathological profile is heavily weighted towards low-risk, such that few (if any) would have a RSPC of $>10 \%$.

Our evidence for the association between RS\% and treatment recommendation relies on observed increasing proportions of patients with recommendations for chemotherapy usage in the three ascending RS\%-assigned risk categories (16.1, 55.1, 92.3\%, respectively). Clinical recommendations of this type do not rely on any single parameter alone, but on a synthesis of multiple observations about tumour biology, clinical presentation, patient co-morbidities and other factors and that the treating clinician and/or the multi-disciplinary team amalgamate to arrive at their final decision regarding treatment recommendations. Countering this as the evidence presented in a recently published retrospective study conducted in the USA taking a look at 431 patients who had their RS result integrated into a multivariable regression analysis together with clinicopathological features. The study found RS indicative of intermediate- or high-risk was the single most influential factor indicating likelihood of chemotherapy use [16].
Currently, Oncotype DX is the only molecular prognostic profiling tool recommended for use in the NHS in England and Wales, but elsewhere the availability of test is broader. For example, American Society of Clinical Oncologists has recently endorsed the use of MammaPrint (agendia NV) for node-negative patients clinically indicated to be at high-risk [17].

There are some limitations to the study presented here and conclusions drawn from its analysis should be viewed with some caution. The sample size is relatively small although the data were largely consistent between the 4 centres despite some differences on the characteristics of recruited patients. We have not been able to present any outcome data; to determine fully the clinical validity of the results, patients would need to be randomised to management according to the 2 scoring methods. All prognostic indices that rely on assessment of clinicopathologic criteria such as grade are potentially subject to confounding intraobserver variability, and this may be a particular problem where strict adherence to standards is not in place. The study's patient population has been defined using NHS England criteria of node-negative and intermediate-risk by NPI or similar, and this is not universally applicable to tested populations in other parts of the world.

\section{Conclusion}

In a clinically relevant patient population judged by standard clinical and pathological features to be at intermediate-risk of disease recurrence, and in England and Wales as defined by NHS England guidelines for Oncotype DX use, RSPC indicates that substantially fewer patients are at lowrisk and substantially more are at high-risk of DR when compared to RS\%. Since RSPC produces a superior estimate of risk (but not necessarily of chemosensitivity), it presents a standard option to use in preference to the RS\% when decisions about chemotherapy recommendation are being made on the basis of a patient's risk of DR. The relatively small sample size and absence of outcome data indicate that conclusions should be viewed with some caution and require further validation by additional studies.

Acknowledgements The authors would like to acknowledge the contributions of Riley Payne who provided data used to identify RMH study patients.

Funding This work was supported by the Royal Marsden National Institute of Health Research Biomedical Research Centre.

\section{Compliance with ethical standards}

Conflict of interest MD declares that he has received lecture fees from Myriad. All remaining authors declare that they have no conflict of interest. 
Ethical approval All procedures performed in studies involving human participants were in accordance with the ethical standards of the institutional research committees and with the 1964 Helsinki declaration and its later amendments or comparable ethical standards.

Research involving animal participants This article does not contain any studies with animals performed by any of the authors.

Open Access This article is distributed under the terms of the Creative Commons Attribution 4.0 International License (http://crea tivecommons.org/licenses/by/4.0/), which permits unrestricted use, distribution, and reproduction in any medium, provided you give appropriate credit to the original author(s) and the source, provide a link to the Creative Commons license, and indicate if changes were made.

\section{References}

1. WHO (2017) Cancer prevention and control. http://www.who.int/ cancer/detection/breastcancer/en/index $1 . h t m l$

2. CRUK (2016) Cancer Research UK: Cancer statistics for the UK. http://www.cancerresearchuk.org/health-professional/cancer-sta tistics. 2016

3. Quaresma M, Coleman MP, Rachet B (2015) 40-year trends in an index of survival for all cancers combined and survival adjusted for age and sex for each cancer in England and Wales, 1971-2011: a population-based study. Lancet 385(9974):1206-1218. doi:10.1016/S0140-6736(14)61396-9

4. Blamey RW, Davies CJ, Elston CW, Johnson J, Haybittle JL, Maynard PV (1979) Prognostic factors in breast cancer-the formation of a prognostic index. Clin Oncol 5(3):227-236

5. Coates AS, Winer EP, Goldhirsch A, Gelber RD, Gnant M, Piccart-Gebhart M, Thurlimann B, Senn HJ, Panel M (2015) Tailoring therapies-improving the management of early breast cancer: St Gallen International expert consensus on the primary therapy of early breast cancer 2015. Ann Oncol 26(8):1533-1546. doi:10.1093/annonc/mdv221

6. NICE (2013) Gene expression profiling and expanded immunohistochemistry tests for guiding adjuvant chemotherapy decisions in early breast cancer management (DG10). https://www.nice. org.uk/guidance/dg10/chapter/About-this-guidance. 2016

7. Tang G, Cuzick J, Costantino JP, Dowsett M, Forbes JF, Crager M, Mamounas EP, Shak S, Wolmark N (2011) Risk of recurrence and chemotherapy benefit for patients with node-negative, estrogen receptor-positive breast cancer: recurrence score alone and integrated with pathologic and clinical factors. J Clin Oncol 29(33):4365-4372. doi:10.1200/JCO.2011.35.3714

8. Paik S, Shak S, Tang G, Kim C, Baker J, Cronin M, Baehner FL, Walker MG, Watson D, Park T, Hiller W, Fisher ER, Wickerham DL, Bryant J, Wolmark N (2004) A multigene assay to predict recurrence of tamoxifen-treated, node-negative breast cancer. N Engl J Med 351(27):2817-2826. doi:10.1056/NEJMoa041588

9. Dowsett M, Cuzick J, Wale C, Forbes J, Mallon EA, Salter J, Quinn E, Dunbier A, Baum M, Buzdar A, Howell A, Bugarini R,
Baehner FL, Shak S (2010) Prediction of risk of distant recurrence using the 21-gene recurrence score in node-negative and node-positive postmenopausal patients with breast cancer treated with anastrozole or tamoxifen: a TransATAC study. J Clin Oncol 28(11):1829-1834. doi:10.1200/JCO.2009.24.4798

10. GHI Oncotype DX tools for genomic education (2017). https:// tools.genomichealth.com/Tools/ToolsStart.aspx

11. Todd JH, Dowle C, Williams MR, Elston CW, Ellis IO, Hinton CP, Blamey RW, Haybittle JL (1987) Confirmation of a prognostic index in primary breast cancer. Br J Cancer 56(4):489-492

12. Paik S, Tang G, Shak S, Kim C, Baker J, Kim W, Cronin M, Baehner FL, Watson D, Bryant J, Costantino JP, Geyer CE Jr, Wickerham DL, Wolmark N (2006) Gene expression and benefit of chemotherapy in women with node-negative, estrogen receptor-positive breast cancer. J Clin Oncol 24(23):3726-3734. doi:10.1200/JCO.2005.04.7985

13. Albain KS, Barlow WE, Shak S, Hortobagyi GN, Livingston RB, Yeh IT, Ravdin P, Bugarini R, Baehner FL, Davidson NE, Sledge GW, Winer EP, Hudis C, Ingle JN, Perez EA, Pritchard KI, Shepherd L, Gralow JR, Yoshizawa C, Allred DC, Osborne CK, Hayes DF, Breast Cancer Intergroup of North A (2010) Prognostic and predictive value of the 21-gene recurrence score assay in postmenopausal women with node-positive, oestrogen-receptor-positive breast cancer on chemotherapy: a retrospective analysis of a randomised trial. Lancet Oncol 11(1):55-65. doi:10. 1016/S1470-2045(09)70314-6

14. Early Breast Cancer Trialists' Collaborative G, Peto R, Davies C, Godwin J, Gray R, Pan HC, Clarke M, Cutter D, Darby S, McGale P, Taylor C, Wang YC, Bergh J, Di Leo A, Albain K, Swain S, Piccart M, Pritchard K (2012) Comparisons between different polychemotherapy regimens for early breast cancer: meta-analyses of long-term outcome among 100,000 women in 123 randomised trials. Lancet 379(9814):432-444. doi:10.1016/ S0140-6736(11)61625-5

15. Sparano JA, Gray RJ, Makower DF, Pritchard KI, Albain KS, Hayes DF, Geyer CE Jr, Dees EC, Perez EA, Olson JA Jr, Zujewski J, Lively T, Badve SS, Saphner TJ, Wagner LI, Whelan TJ, Ellis MJ, Paik S, Wood WC, Ravdin P, Keane MM, Gomez Moreno HL, Reddy PS, Goggins TF, Mayer IA, Brufsky AM, Toppmeyer DL, Kaklamani VG, Atkins JN, Berenberg JL, Sledge GW (2015) Prospective validation of a 21-gene expression assay in breast cancer. N Engl J Med 373(21):2005-2014. doi:10.1056/ NEJMoa1510764

16. Rutter CE, Yao X, Mancini BR, Aminawung JA, Chagpar AB, Saglam O, Hofstatter EW, Abu-Khalaf M, Gross CP, Evans SB (2016) Influence of a 21-gene recurrence score assay on chemotherapy delivery in breast cancer. Clin Breast Cancer 16(1):59-62. doi:10.1016/j.clbc.2015.09.008

17. Krop I, Ismaila N, Andre F, Bast RC, Barlow W, Collyar DE, Hammond ME, Kuderer NM, Liu MC, Mennel RG, Van Poznak C, Wolff AC, Stearns V (2017) Use of biomarkers to guide decisions on adjuvant systemic therapy for women with earlystage invasive breast cancer: american society of clinical oncology clinical practice guideline focused update. J Clin Oncol 35(24):2838-2847. doi:10.1200/JCO.2017.74.0472 